\title{
ToxicBlend: Virtual Screening of Toxic Compounds with Ensemble Predictors
}

\author{
Mikhail Zaslavskiy, Simon Jégou, Eric W. Tramel, \& Gilles Wainrib \\ Owkin, Inc. \\ New York City, New York
}

June 13, 2018

\begin{abstract}
Timely assessment of compound toxicity is one of the biggest challenges facing the pharmaceutical industry today. A significant proportion of compounds identified as potential leads are ultimately discarded due to the toxicity they induce. In this paper, we propose a novel machine learning approach for the prediction of molecular activity on ToxCast targets. We combine extreme gradient boosting with fullyconnected and graph-convolutional neural network architectures trained on QSAR physical molecular property descriptors, PubChem molecular fingerprints, and SMILES sequences. Our ensemble predictor leverages the strengths of each individual technique, significantly outperforming existing state-of-the art models on the ToxCAST and Tox21 toxicity-prediction datasets. We provide free access to molecule toxicity prediction using our model at http://toxicblend.owkin.com.
\end{abstract}

Keywords - Toxicity prediction, Ensemble models, Virtual screening

\section{Introduction}

In silico models for molecular activity prediction have become critical for drug discovery and other biotechnology industries 1. These methods prove very important for the prediction of intrinsic chemical properties (such as solubility [2 or acidity constant [3]) or target affinity prediction [4, including potential toxicity effects, a crucial step in the development of new drugs.

Over the years, two complementary computational approaches have been developed to achieve this goal. The first approach, physical modeling, focuses on computational simulations of physical molecular interactions. This approach requires the knowledge about the nature of the target and its well-described 3D structure. Subsequently, significant computational resources are required for running the simulation, as the space of possible configurations is vast. While this approach provides accurate descriptions of molecular dynamics, these two requirements prohibit large-scale deployment of this technique to ameliorate the costs associated with physical high-throughput screening (HTS).

In the second approach, one instead seeks to construct a predictive model learned from a large set of experimental measurements via statistical modeling and machine learning. While the training of such models may be costly and time-consuming, their usefulness in predicting the toxicity of novel compounds can be observed in their scalability, as they allow for virtual screening of vast chemical databases, even when structural information is not available.

One of the major limiting factors in the successful deployment of advanced machine learning methods for toxicity prediction is the requirement for large datasets from which to train such complex models. To foster further development of machine learning techniques, multiple public initiatives [5] have been launched to compile datasets with wide coverage of compounds and their properties. One such project is the ToxCAST project 2 supported by the U.S. Environmental Protection Agency (EPA), which offers the scientific community unprecedented datasets of observed in vitro interactions between thousands of small molecules and known toxicity-related targets. The TOxCAST project seeks to better understand the underlying mechanisms of action of various toxic chemicals, and to provide support for predictive models that could be used to prioritize potential toxicants for in vivo testing.

As witnessed in the Tox21 Challenge, a spin-off of the ToxCAST project organized in 2014 [6], techniques based around deep neural networks (DNNs), are able to provide state-of-the-art performance for virtual toxicity screening $[7-12]$. The winning solution of [13] utilized an ensemble of various classifiers built upon many thousands of chemical descriptors. 
Subsequently, a wide range of new descriptors and chemical fingerprints have been used in conjunction with machine learning approaches for the prediction of various properties of molecules 14 19. More recently, several papers have explored methods such as graph convolutional networks (GCNs) [20 23], bypassing conventional chemical fingerprinting and working directly on the molecular graph structure, leading to state-of-the-art performance across a wide range of molecular prediction tasks, as reported in 24. Such approaches draw inspiration from convolutional neural networks for images, even directly from drawings of molecules as in 25].

In this work, we take a pragmatic approach, proposing a systematic ensembling method for virtual toxicity screening. We combine various molecular descriptors with three different predictive modeling approaches: gradient tree boosting, DNNs, and GCNs. The aim of this work is to establish state-of-theart predictors for 617 TOxCAST targets as well as twelve Tox21 and to provide the scientific community with a ready-to-use prediction model for toxicity pre-screening through our web server ToxicBlend http: //toxicblend.owkin.com.

To show the advantage of the proposed model, we report objective results against baseline virtual toxicity screening methods such as those implemented in DeepChem 24 using cross-validation performed via three different splitting scenarios: by index, by scaffold, as well as by random selection.

\section{Materials}

\subsection{Datasets}

In our experiments, we use the Tox21 and ToxCAST datasets obtained from the DeepChem package 24 . The Tox21 dataset was constructed for the Toxicity prediction challenge [6], organized by the National Center for Advancing Translational Sciences (NCATS) at the National Institutes of Health (NIH). The ToxCAsT dataset, constructed for the Toxcicity Forecaster program, is much borader in scope, containing many hundreds of toxicity assay targets.

Tox21. The Tox21 dataset consists of toxicity assays conducted over 8,000 chemical compounds on twelve different targets. Seven assays represent the response of five nuclear receptors: the androgen and estrogen receptors, for both the general and ligand binding domain, as well as the aryl hydrocarbon, glitazone, and aromatase receptors. The five remaining assays measure the following cell stress responses: oxidative stress (ARE), genetic stress (ATAD5), heat shock response (HSE), mitochondrial function disruption (MMP), as well as DNA damage and other miscellaneous cellular stress (p53).

ToxCast. The ToxCAST dataset is similar in nature to Tox21, but with a much wider range of performed toxicity assays. Table 1 provides the distribution of ToxCAsT assays per target family type. In total, there are 617 assays measuring the toxicity effects of more than 8,000 chemical compounds.

\begin{tabular}{lrlr}
\hline Family & Count & Family & Count \\
\hline Nuclear Receptor & 115 & Oxidoreductase & 12 \\
DNA Binding & 77 & Kinase & 11 \\
Background Measurement & 62 & Ion Channel & 9 \\
GPCR & 55 & Phosphatase & 7 \\
Cytokine & 55 & Esterase & 6 \\
Cell Cycle & 51 & Transferase & 5 \\
Cell Morphology & 30 & Growth Factor & 4 \\
CYP & 26 & Misc. Protein & 3 \\
Cell Adhesion Molecules & 21 & Hydrolase & 3 \\
Protease & 17 & Protease Inhibitor & 2 \\
Steroid Hormone & 14 & & \\
Transporter & 12 & Not defined & 20 \\
\hline
\end{tabular}

Table 1: Number of toxicity assays performed per target family for the ToxCAST dataset. 


\subsection{Dataset Stratifications}

To estimate the performance of individual models, as well as the final blended predictor, we perform three types of cross-validation experiments based on random, scaffold and index splits, as in [24]. For random splitting, all ligands were divided randomly into three groups: training (80\%), validation, (10\%) and test sets $(10 \%)$. When using scaffold splitting, all ligands were grouped according to their scaffolds, such that ligands with the same scaffold were always assigned to the same set (training, validation, or test). Index based splitting follows the natural order of rows in the dataset, it uses first $80 \%$ rows as the training set and the following $10 \%$ and $10 \%$ as validation and test sets.

\section{Molecular Feature Extraction}

The final predictive model is built on top of several individual machine learning models trained on various feature subsets. We tested different molecular fingerprint families such as PubChem fingerprints, extended connectivity fingerprints [26], DFS fingerprints [27], molprint 2D fingerprints 28], topological autocorrelation keys [29, Shannon entropy descriptors [30, and several molprint 3D fingerprints implemented in java Compound Mapper [31]: geometrical autocorrelation keys, 3D atom triplets 32] and 3D molprint-like fingerprints.

Another, completely different, source of information about molecules was derived using computational models for the estimation of various molecular properties ranging from simple (e.g. total molecular mass, number of atoms, number of chemical bonds, etc.), to complex, such as molecular solubility and polarity, which are known to be directly related to molecular activity potential [33. In addition to these two groups of descriptors, we also tested the capacity of machine learning models to work directly on the molecule SMILES, themselves. Although SMILES representations can be seen to be overly complicated for ML techniques, compared to molecular graphs for instance, we are able to show that models trained directly on SMILES features can be competitive with those trained on molecular fingerprint representations for both Tox21 and ToxCAst. For the individual models we train, we utilize physical ligand descriptors, PubChem molecular fingerprints, and SMILES $n$-grams, which we describe in detail below.

Physical Ligand Descriptors (PLD). The first set of 47 features correspond to various QSAR molecular descriptors computed using the chemistry development kit 34]. These descriptors represent physico-chemical properties of molecules such as mass, atom count, solubility, and polarity, among others. We refer the reader to Sec. A.1 for a complete list of extracted features.

PubChem Molecular Fingerprints (PCFP). The PubChem fingerprint is a 881-bit descriptor indicating the presence or absence of a predefined list of 881 molecular subgraphs and subgraph families within a given compound 1 .

SMILES $n$-grams (SNG). SMILES $n$-gram features consist of counts of SMILES sub-sequences, up to $n$ characters. In our experiments, we used $n \in\{3,4\}$. These features can be seen as a simplified version of molecular-graph fingerprints, where fingerprints are defined by the canonical SMILES representation 34 .

\section{Toxicity Prediction Models}

We tested every molecular feature subset in conjunction with the a few machine learning models trained in a multi-task setting, namely: extreme gradient boosting trees (Xgboost) [35], DNNs [36], as well as GCNs as implemented in the DeepChem package [24]. All model hyper-parameters were optimized using the validation set. In addition to testing the prediction performance of our final blended model using these models, we also attempted to blend additional baseline classifiers such as Lasso, ride regression, random forests 37], and K-nearest neighbors. However, we found empirically that these simple methods did not provide a significant improvement in toxicity prediction accuracy.

\subsection{Multi-task extreme gradient boosting}

Our multi-task gradient boosting model is trained on an entire dataset simultaneously by stacking all taskspecific data together and adding a descriptor representing the identity of the corresponding task. This representation allows the gradient boosting algorithm to leverage information from different tasks and to

\footnotetext{
${ }^{1} \mathrm{ftp}: / /$ ftp.ncbi.nlm.nih.gov/pubchem/specifications/pubchem_fingerprints.txt
} 
learn characteristics indicating ligand toxicity across multiple tasks. At the same time, the algorithm can build task specific rules by combining the task identity descriptor with the corresponding ligand features.

To train a gradient boosting model, we used the Xgboost package 35]. For each cross-validation iteration, we optimized three hyper-parameters using the held-out validation split: i) the number of rounds, up to $20 \mathrm{k}$ rounds with an early stopping triggered by validation performance, ii) the depth, up to 20 , and iii) the shrinkage parameter, which was selected from the set of values $\{1 \mathrm{e}-2,5 \mathrm{e}-3,1 \mathrm{e}-3,5 \mathrm{e}-4\}$.

\subsection{Multi-task neural networks}

Deep learning methods have proved to be very efficient for the toxicity prediction task were essential in the winning methods of the Tox21 challenge [38,39. Multi-task neural networks are particularly effective for molecular inputs, as shown in 14. Instead of building $T$ individual networks to tackle $T$ different tasks, we use a single network with $T$ outputs. This construction permits the use all available information on the input molecule within a single network, enriching intermediate representations.

As in 38, all neural networks architectures were manually optimized for each evaluated dataset and each set of molecular features. In contrast with the single-layer multi-task NN demonstrated in [40], we optimized architectures over a set of shallow NN models consisting of $L=\{2,3\}$ layers, each of which may contain $M=\{256,516,1024\}$ neurons. All networks use ReLU activations 2 , with dropout regularization applied to each layer at a rate of 0.5 to prevent over-fitting. In the case of NNs trained on PCFP inputs, we used a dropout rate of 0.1 in order to account for the extreme sparsity of these features. All networks were trained to minimize the average binary cross-entropy over the set of $T$ targets,

$$
\mathcal{L}(\mathbf{y}, \widetilde{\mathbf{y}} ; \Theta) \triangleq \frac{\sum_{t=1}^{T} \sum_{i=1}^{N} \delta\left(1+y_{t i}\right) \times\left[y_{t i} \log \widetilde{y}_{t i}+\left(1-y_{t i}\right) \log \left(1-\widetilde{y}_{t i}\right)\right]}{\sum_{t=1}^{T} \sum_{i=1}^{N} \delta\left(1+y_{t i}\right)},
$$

where $\delta(\cdot)$ is the Dirac delta function such that $\delta(0)=1$ and 0 everywhere else, and $\widetilde{\mathbf{y}}=\left[\widetilde{y}_{t i} ; \forall t, i\right]$ are the labels inferred from the NN with parameters $\Theta$ for the given mini-batch training samples. The groundtruth labels $\mathbf{y}=\left[y_{t i} ; \forall t, i\right]$ are assigned 1 if the ligand $i$ is active for the target $t, 0$ if it is inactive, and -1 if the information is not available. The value $N$ is the mini-batch size, $N=512$ for our experiments. The model parameters $\Theta$ were optimized to minimize (1) using Adam [41. We used early stopping after 25 epochs when no improvement was observed on the loss of the validation set. For our implementation, we use the Keras 42 framework in conjunction with Tensorflow 43 .

\subsection{Graph Convolutional Models}

GCNs [40], can be seen as a more general version of molecular circular fingerprint [44], where fingerprints are learned directly from the dataset. For more details on this approach as applied to molecular data, we refer the reader to 24, 40]. In our experiments, we train GCNs using the DeepChem package [24].

\section{Model Blending}

For state-of-the-art toxicity prediction, we propose a final predictor which is built on top of different models trained on the three feature subgroups we detail at the end of Sec. 3. During experimentation, we found that this approach is more efficient, and less prone to overfitting, compared to a single model trained over all available extracted molecular features.

We built our blended predictor using multi-task gradient boosting with an additional constraint on the monotonous dependency between the individual model predictions and the final combined score [45]. This constraint helps avoid overfitting and also promotes an efficient combination of prediction models, even when the number of training samples is small.

\section{$6 \quad$ Results}

For each of the individual models, validation splits are used for hyper-parameter tuning. Toxicity predictions on the held-out test sets were used for the assessment of final model performance. Our proposed blended predictor was trained using individual model predictions on the validation splits.

Table 2 presents the performance of all individual models, as well as our blended predictor. Here, the presented AUC values are averages over ten independent trials for both the random and scaffold splits. For the index split, only a single trial is used. Of the individual models, Xgboost trained on chemical

${ }^{2} \operatorname{ReLU}(x)=\max (x, 0)$ 

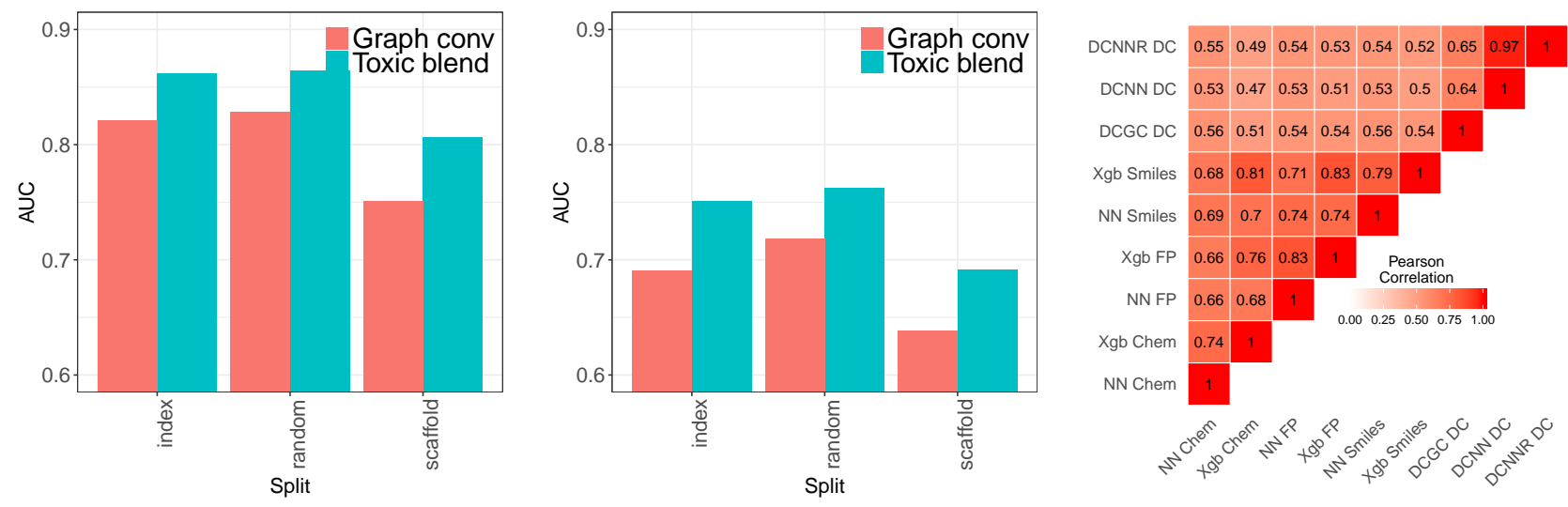

Figure 1: AUC comparison between state-of-the-art toxicity prediction with a single GCN, and the proposed blended ensemble of multiple approaches, shown over different cross-validation split approaches, for the Tox21 (left) and ToxCAST (middle) datasets. Right: Correlation coefficients between individual prediction models in the blended ensemble, averaged over different tasks in the Tox21 dataset.

descriptors provides the best AUC performance on random splits. This result suggests that chemical properties estimated by computational models can provide rich features for accurate toxicity prediction. The performance gap grows when we split ligands by scaffold, indicating that the chemical descriptors better describe cross-scaffold properties important for toxicity prediction. Interestingly, models trained only on SMILES subsequences already provide enough information for machine learning models to generate competitive predictions. Finally, when we combine all methods together using our blended predictor, we observe a performance improvement of 0.5 to 2 AUC points. Fig. 1 1 compares this gain against the bestperforming GCN model from the DeepChem package [24 for both datasets across the tested splitting strategies.

In Fig. 1. we also show the Pearson correlation coefficients calculated between individual models. Low correlation coefficients between the predictions of individual models indicates a diverse pool of predictors from which to build a blended model. Such diversity generally leads to a robust final blend, as the failure of a particular indicator does not correlate with the failure of other indicators. However, this diversity requires a thoughtful construction of the blending method. For example, the high variance between the performance of individual models prevents the use of simple ensembling techniques such as prediction averaging. Concretely, attempting such an averaging for random splits of the Tox21 dataset leads to an average AUC of 0.851: a level of performance worse than the best performing individual model, as shown in Table 2. In the case of random splits on ToxCAST, prediction averaging provides an AUC of 0.754, a result better than the individual models, but still less than our proposed blending approach (0.763).

\begin{tabular}{|c|c|c|c|c|c|c|c|}
\hline \multirow[t]{2}{*}{ Model } & \multirow[t]{2}{*}{ Features } & \multicolumn{3}{|c|}{ Tox21 } & \multicolumn{3}{|c|}{ ToxCAst } \\
\hline & & index & random & scaffold & index & random & scaffold \\
\hline NN & PLD & 0.821 & 0.821 & 0.751 & 0.732 & 0.716 & 0.657 \\
\hline $\mathrm{NN}$ & PCFP & 0.831 & 0.837 & 0.761 & 0.730 & 0.738 & 0.670 \\
\hline $\mathrm{NN}$ & SNG & 0.821 & 0.839 & 0.770 & 0.722 & 0.739 & 0.663 \\
\hline $\mathrm{Xgb}$ & PLD & 0.842 & 0.853 & 0.803 & 0.742 & 0.740 & 0.680 \\
\hline $\mathrm{Xgb}$ & PCFP & 0.846 & 0.852 & 0.773 & 0.731 & 0.738 & 0.660 \\
\hline $\mathrm{Xgb}$ & $\mathrm{SNG}$ & 0.854 & 0.852 & 0.777 & 0.738 & 0.742 & 0.668 \\
\hline DCGC & $\mathrm{DC}$ & 0.821 & 0.829 & 0.751 & 0.690 & 0.718 & 0.639 \\
\hline DCNN & $\mathrm{DC}$ & 0.785 & 0.787 & 0.724 & 0.651 & 0.676 & 0.614 \\
\hline DCNNR & $\mathrm{DC}$ & 0.784 & 0.790 & 0.730 & 0.654 & 0.683 & 0.614 \\
\hline Pred. Avg. & & 0.849 & 0.851 & 0.793 & 0.751 & 0.754 & 0.688 \\
\hline ToxicBlend & & 0.866 & 0.862 & 0.807 & 0.753 & 0.763 & 0.693 \\
\hline
\end{tabular}

Table 2: AUC cross-validation performance scores, averaged over 10 independent random splits for random and scaffold splits. There is only one unique index split, the corresponding value is showed in the table. 

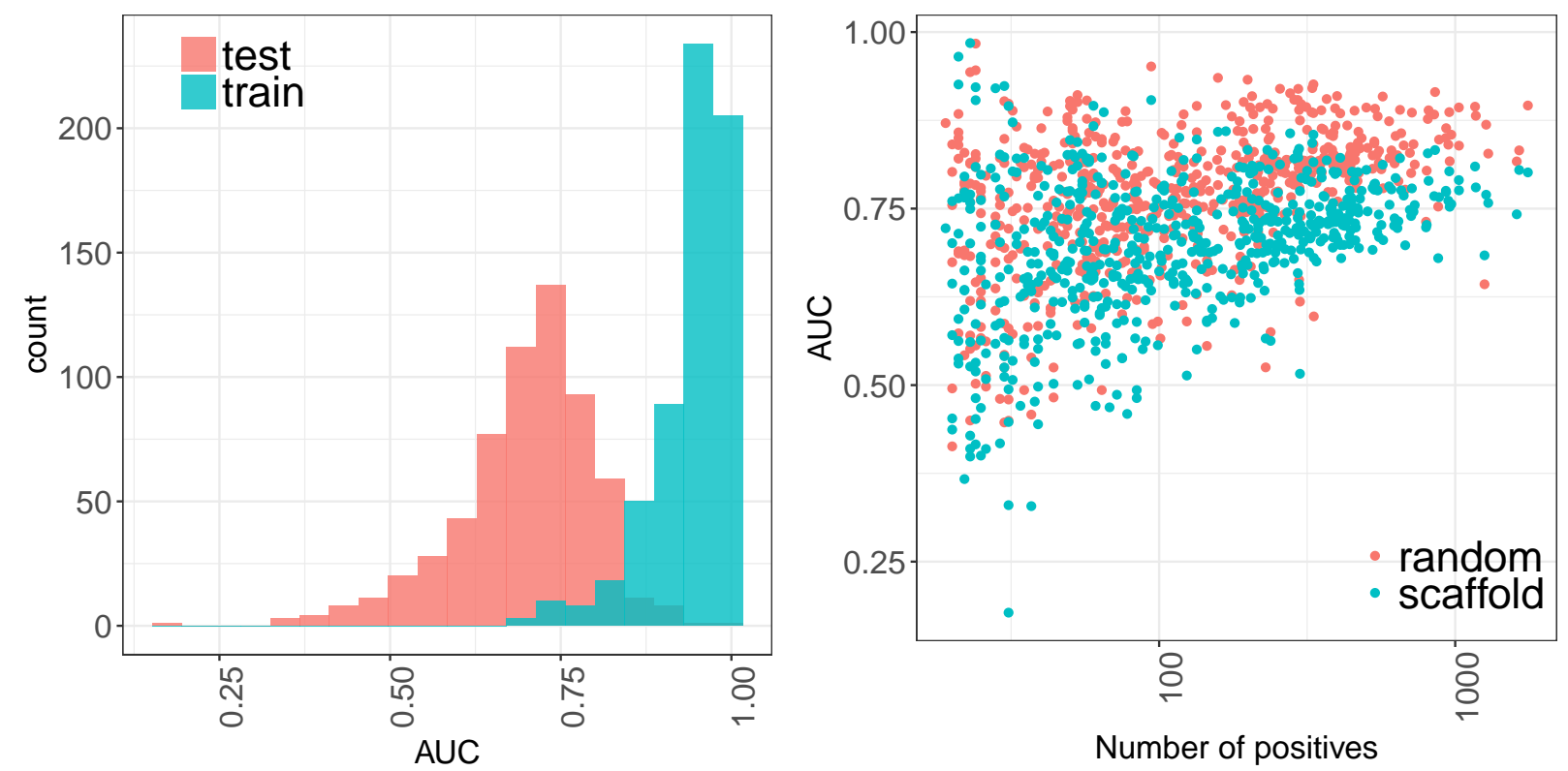

Figure 2: (Left): Distribution of train and test per target AUC scores, scaffold split on ToxCAST dataset. (Right): Number of positive examples versus the target test AUC score in the case of random and scaffold splits.

\subsection{Per-target performance}

The final scores in Tab. 2 are aggregated scores across many different targets, some of which are harder to predict than others. In Fig. 2 (Left), we show the histogram of train and test AUC scores (scaffold split) on ToxCAST dataset. While the training scores never fall below 0.7 , on the test splits there are targets with an average AUC below 0.5, and targets with an AUC above 0.9. We provide a list of the ten hardest and easiest to predict targets for ToxCAST in Suppl. Table A.2 There are several possible physical explanations for such a heterogeneity of performance scores across targets: the capacity of the binding pocket to accept different types of ligands, the level of noise in target specific assays, etc. We identified one important factor in determining per-target performance which arises simply from the data-collection process: the number of positive examples for each target. In Fig. 2 (Right), we show the clear correlation between the number of positive examples for each target plotted against and the per-target AUC achieved by our blended model. Targets with more than 350 positive examples, for instance, never fall below an AUC of 0.64 or 0.69 , for scaffold and random splits, respectively.

\subsection{Ligand feature importance}

In this section we discuss features that were identified as important by DL and Xgboost models. Fig. 3 (Left) shows the relative ligand feature importance defined as the gain computed by gradient boosting trees algorithm.

For neural networks, we used a permutation feature importance technique. For each feature, we randomly shuffled the values associated to this feature and the $k$ most correlated features, and computed the average AUC degradation over 5 folds, for $k=0,5$ and 10. The lower the AUC, the more important the feature.

Table 3 lists the top ten features according to their importance ranking for both chemical and PubChem fingerprint descriptors. Interestingly, many of the most important fingerprint descriptors are related to the size of the molecule, indicating that size characteristics might be a critical indicator for toxicity prediction. When trained on chemical descriptors, the individual Xgboost model prioritizes Broto-Moreau autocorrelation features (ATSpx) built on atomic polarizabilities, Ghose-Crippen molar refractivity (AMR), number of bonds, eigenvalue descriptors based on Burden matrix, Wiener polarity number, and the solvent accessible surface areas of atoms (THSA and TPSA).

\subsection{Reliability of the model}

Why should we trust our model? The cross-validated AUC estimate gives us a global confidence level per target, and for a given molecule, the probability estimated by the model measures the uncertainty of the 

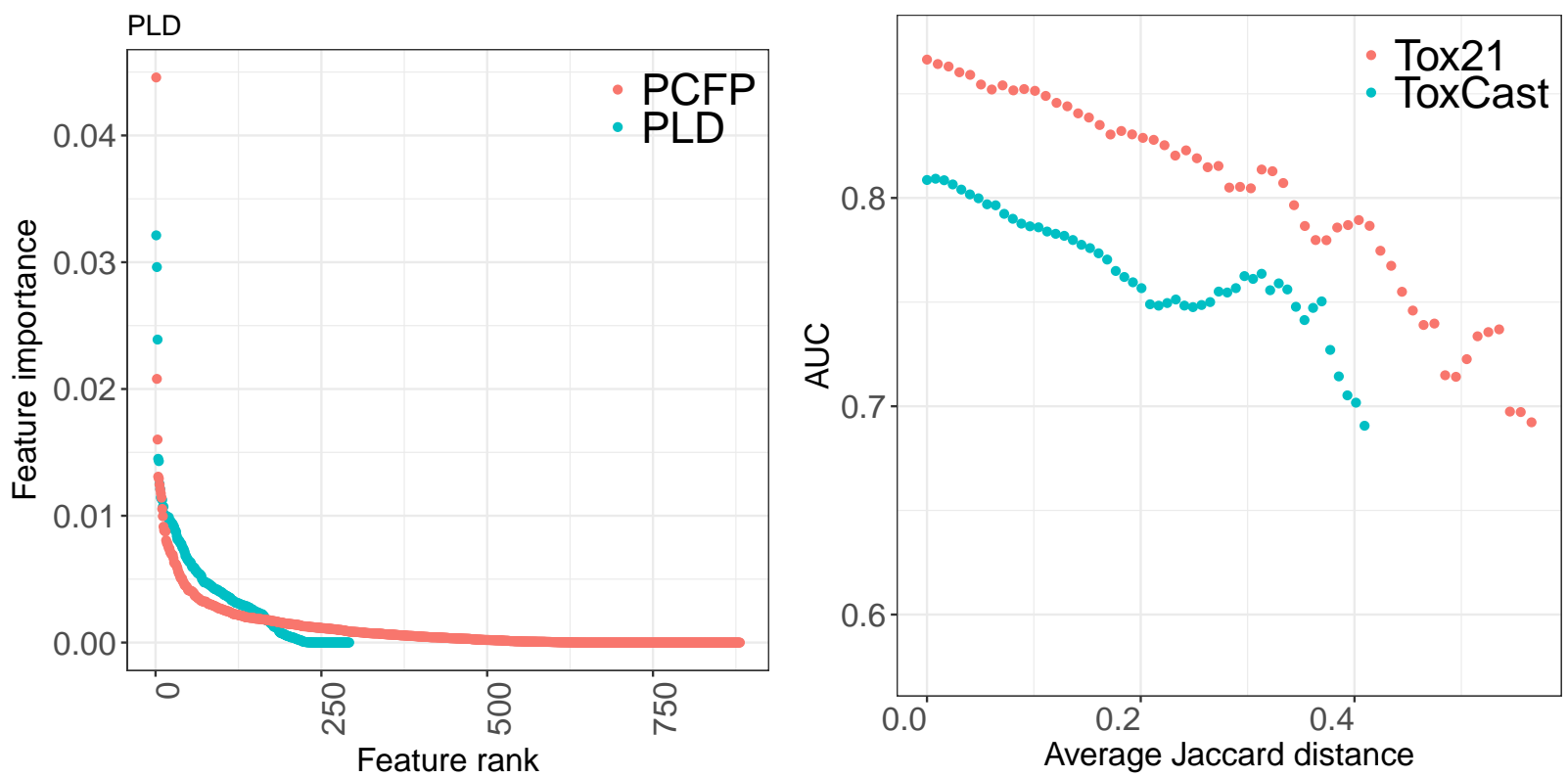

Figure 3: Left: Xgboost gain on ToxCAST dataset for both PLD (left) and PCFP (right) features. Right: Average AUC score against the average Jaccard distance to the training set.

\begin{tabular}{|c|c|c|c|c|}
\hline & \multicolumn{2}{|c|}{ Chem } & \multicolumn{2}{|c|}{ PCFP } \\
\hline & $X g b$ & $N N$ & $X g b$ & $N N$ \\
\hline 1. & ATSp3 & HybRatio & $\geq 1 \mathrm{~N}$ & $\geq 16 \mathrm{C}$ \\
\hline 2. & ATSp4 & C1SP2 & O-C-C-C-C-C-C-C & $\geq 1 \mathrm{Sn}$ \\
\hline 3. & AMR & Wnu1.unity & $\geq 32 \mathrm{C}$ & $\geq 2$ any ring size 6 \\
\hline 4. & $\mathrm{nB}$ & ATSm4 & $\geq 16 \mathrm{O}$ & $\overline{\mathrm{C}}-\mathrm{C}-\mathrm{C}-\mathrm{C}-\mathrm{C}-\mathrm{C}-\mathrm{C}-\mathrm{C}$ \\
\hline 5. & BCUTp.1l & VP.3 & $\begin{array}{l}\geq 2 \text { unsaturated non-aromatic } \\
\text { heteroatom-containing ring size } 6\end{array}$ & $\begin{array}{l}\geq 2 \text { unsaturated non-aromatic } \\
\text { carbon-only ring size } 6\end{array}$ \\
\hline 6. & ATSm1 & MDEC.34 & $\geq 8 \mathrm{O}$ & $\geq 3$ any ring size 6 \\
\hline 7. & WPOL & Weta1.unity & $\mathrm{O}=\mathrm{C}-\mathrm{C}-\mathrm{O}$ & $\geq 1 \mathrm{Hg}$ \\
\hline 8. & THSA & ATSp4 & $\mathrm{C}(\sim \mathrm{N})(: \mathrm{C})$ & $\mathrm{Sc} 1 \mathrm{c}(\mathrm{Cl}) \operatorname{cccc} 1$ \\
\hline 9. & TPSA & ATSp1 & $\geq 4 \mathrm{Cl}$ & $\mathrm{SC} 1 \mathrm{C}(\mathrm{Cl}) \mathrm{CCCC} 1$ \\
\hline 10. & ATSm2 & ATSp3 & $\geq 8 \mathrm{Cl}$ & $\mathrm{S}(-\mathrm{O})(=\mathrm{O})$ \\
\hline
\end{tabular}

Table 3: Ten most important chemical properties and PubChem fingerprint features according to individual Xgboost and neural network classifiers trained on the ToxCAST dataset.

prediction. However, both of these measures were obtained using the training distribution, which may not be representative of the distribution of molecules at test time.

To measure the distance from a new molecule $\mathbf{x}$ to the training distribution $X_{\text {train }}$, we use the average Jaccard distance on the $k=5$ nearest neighbors in the fingerprint space,

$$
d\left(\mathbf{x}, X_{\text {train }}\right)=\frac{1}{k} \sum_{\mathbf{x}^{\prime} \in N_{\text {train }}(\mathbf{x}, k)} 1-\frac{\left|\mathbf{x} \cap \mathbf{x}^{\prime}\right|}{\left|\mathbf{x} \cup \mathbf{x}^{\prime}\right|} .
$$

For each target, we compute the AUC score of the test molecules above a given distance threshold. Averaged over 10 test folds (random splits), and all possible targets, we observed a that a higher distance threshold leads to a lower AUC score for both the Tox21 and the ToxCAsT datasets, as shown in Figure 3 (Right). This indicates that the model is less accurate on test molecules far from the distribution of training molecules.

We also observe the same trend between the AUC score and the complexity of the test molecule, expressed as the count of ones in the fingerprint vector. We hypothesize that the higher diversity of complex molecules requires the model to be trained on more data to be accurate. 


\subsection{Experimental variability}

Many biological experiments are subject to considerable variability, and it is often impossible to build a perfect prediction for the simple reason that the experiments themselves are not $100 \%$ reproducible. Thus, it is useful to estimate the best-case toxicity prediction performance. The raw Tox 21 data, as published on NIH website ${ }^{3}$, contains some instances of multiple measurements for the same molecule/target couples. From these measurements, we observe significant heterogeneity between targets in terms of the result reproducibility. For example, $11 \%$ of repeated measurements corresponding to NR-ER contain contradictory results, while NR-PPAR-gamma have only $1 \%$ of such contradictory measurements. If we suppose that homogeneous repeated measurements are likely to stay as they are, and contradictory measurements correspond to a random flip, then an average (across all Tox21 targets) AUC score that we could get by predicting results of an experiment with an additional experiment would be 0.942 .

\section{Discussion}

In this paper, we present a new machine learning method for the prediction of molecule activities in Tox21 and ToxCAST assays, namely, an approach to combine multiple classifiers based on different molecular descriptors. The new approach is implemented as a web server and can be accessed at http: //toxicblend.owkin.com and can be used to predict the toxicity of chemical compounds from their SMILES.

One of the main conclusions of our work is that it is beneficial to use known molecular descriptors that were identified in the past as potentially important for various biochemical properties of molecules, and it is important to combine information sources of different kinds to improve the quality of predictions. An important direction for our future work is the incorporation of target-specific information, such as target structure, in the prediction model. In experimentation (data not shown), we also used physical docking energy scores computed by Smina 46] to attempt to boost the final prediction performance on Tox21 where seven out of twelve targets have known structures. However, it did not lead to any significant improvements. One possible alternative strategy to simple energy scoring is to use more elaborate structure-based descriptors representing target pockets, and other target characteristics, and let ML algorithms determine connections between ligand and target features. Such an approach would demand high target diversity, thus an important direction for future work would be to build an extensive representation for TOXCAST targets in order to evaluate the potential gain from such target descriptors. Mining scientific literature is another potentially interesting source of information. This kind of approach can be seen as an automatic way to construct efficient molecular descriptors.

To remain comparable with existing methods developed for toxicity prediction, we worked only with the Tox21 and ToxCAST datasets, but an important future step would be to use external data sources such as $C h E M B L$ for general ligand activity, BindingDB for structural information, or other existing datasets on molecule toxicity such as RTECS.

${ }^{3}$ https://tripod.nih.gov/tox 21 


\section{A Appendix}

1. ALOGPDescriptor

2. APolDescriptor

3. AminoAcidCountDescriptor

4. AromaticAtomsCountDescriptor

5. AromaticBondsCountDescriptor

6. AtomCountDescriptor

7. AutocorrelationDescriptorCharge

8. AutocorrelationDescriptorMass

9. AutocorrelationDescriptorPolarizability

10. BCUTDescriptor

11. BPolDescriptor

12. BondCountDescriptor

13. CPSADescriptor

14. CarbonTypesDescriptor

15. ChiChainDescriptor

16. ChiClusterDescriptor

17. ChiPathClusterDescriptor

18. ChiPathDescriptor

19. EccentricConnectivityIndexDescriptor

20. FMFDescriptor

21. FragmentComplexityDescriptor

22. GravitationalIndexDescriptor

23. HBondAcceptorCountDescriptor

24. HBondDonorCountDescriptor
25. HybridizationRatioDescriptor

26. KappaShapeIndicesDescriptor

27. KierHallSmartsDescriptor

28. LargestChainDescriptor

29. LargestPiSystemDescriptor

30. LengthOverBreadthDescriptor

31. LongestAliphaticChainDescriptor

32. MDEDescriptor

33. MannholdLogPDescriptor

34. MomentOfInertiaDescriptor

35. PetitjeanNumberDescriptor

36. PetitjeanShapeIndexDescriptor

37. RotatableBondsCountDescriptor

38. RuleOfFiveDescriptor

39. TPSADescriptor

40. VABCDescriptor

41. VAdjMaDescriptor

42. WHIMDescriptor

43. WeightDescriptor

44. WeightedPathDescriptor

45. WienerNumbersDescriptor

46. XLogPDescriptor

47. ZagrebIndexDescriptor

Table A.1: List of physical descriptors 


\begin{tabular}{|c|c|c|c|c|c|}
\hline \multicolumn{3}{|l|}{ Easiest Targets } & \multicolumn{3}{|l|}{ Hardest Targets } \\
\hline Target & Test & Train & Target & Test & Train \\
\hline TOX21_AutoFluor_HEPG2_Media_green & 0.98 & 1.00 & NCCT_TPO_GUA_dn & 0.18 & 0.88 \\
\hline NVS_NR_rMR & 0.97 & 0.93 & ATG_Oct_MLP_CIS_dn & 0.33 & 0.87 \\
\hline NVS_GPCR_hAT1 & 0.93 & 0.99 & NVS_ENZ_hPTEN & 0.33 & 0.88 \\
\hline NVS_NR_hRAR_Antagonist & 0.92 & 0.97 & NVS_ENZ_hMMP7 & 0.37 & 0.81 \\
\hline TOX21_AutoFluor_HEPG2_Cell_green & 0.92 & 1.00 & NVS_ENZ_hAurA & 0.40 & 0.95 \\
\hline NVS_GPCR_rr & 0.92 & 0.93 & BSK_KF3CT_MCP1_up & 0.40 & 0.75 \\
\hline TOX21_ESRE_BLA_ch1 & 0.90 & 1.00 & NVS_LGIC_hNNR_NBungSens & 0.41 & 0.80 \\
\hline TOX21_PPARg_BLA_Agonist_ch1 & 0.90 & 1.00 & ATG_PXR_TRANS_dn & 0.41 & 0.94 \\
\hline APR_HepG2_NuclearSize_24h_dn & 0.90 & 0.99 & BSK_KF3CT_IP10_up & 0.42 & 0.71 \\
\hline NVS_ADME_rCYP2C12 & 0.90 & 0.97 & ATG_TA_CIS_dn & 0.42 & 0.81 \\
\hline
\end{tabular}

Table A.2: Top ten easiest and hardest to predict targets in ToxCAST dataset.

\section{References}

[1] Y. Tanrikulu, B. Krüger, and E. Proschak, "The holistic integration of virtual screening in drug discovery," Drug Discovery Today, vol. 18, no. 7, pp. 358-364, 2013.

[2] D. S. Palmer, N. M. O'Boyle, R. C. Glen, and J. B. Mitchell, "Random forest models to predict aqueous solubility," Journal of chemical information and modeling, vol. 47, no. 1, pp. 150-158, 2007.

[3] M. Rupp, R. Körner, and I. Tetko, Predicting the pKa of Small Molecules, vol. 14. 042011.

[4] P. M.-U. Ung, W. Song, L. Cheng, X. Zhao, H. Hu, L. Chen, and A. Schlessinger, "Inhibitor discovery for the human glut1 from homology modeling and virtual screening," ACS Chemical Biology, vol. 11, pp. 1908-1916, 072016.

[5] A. Subramanian, R. Narayan, S. M. Corsello, D. D. Peck, and T. E. N. et al, "A next generation connectivity map: L1000 platform and the first 1,000,000 profiles.," vol. 171, no. 6, pp. 1437-1452, 2017.

[6] R. Huang and M. Xia, "Tox21 challenge to build predictive models of nuclear receptor and stress response pathways as mediated by exposure to environmental toxicants and drugs," Frontiers in Environmental Science, vol. 5, p. 3, 2017.

[7] A. Koutsoukas, J. St Amand, M. Mishra, and J. Huan, "Predictive toxicology: Modeling chemical induced toxicological response combining circular fingerprints with random forest and support vector machine. front," Tox21 Challenge to Build Predictive Models of Nuclear Receptor and Stress Response Pathways as Mediated by Exposure to Environmental Toxicants and Drugs, vol. 4, no. 11, p. 85, 2017.

[8] R. Huang and M. Xia, "Tox21 challenge to build predictive models of nuclear receptor and stress response pathways as mediated by exposure to environmental toxicants and drugs," Frontiers in Environmental Science, vol. 5, p. 3, 2017.

[9] K. Ribay, M. T. Kim, W. Wang, D. Pinolini, and H. Zhu, "Predictive modeling of estrogen receptor binding agents using advanced cheminformatics tools and massive public data," Frontiers in environmental science, vol. 4, 2016.

[10] M. N. Drwal, V. B. Siramshetty, P. Banerjee, A. Goede, R. Preissner, and M. Dunkel, "Molecular similarity-based predictions of the tox21 screening outcome," Tox21 Challenge to Build Predictive Models of Nuclear Receptor and Stress Response Pathways as Mediated by Exposure to Environmental Toxicants and Drugs, p. 62, 2017.

[11] F. Stefaniak, "Prediction of compounds activity in nuclear receptor signaling and stress pathway assays using machine learning algorithms and low-dimensional molecular descriptors. front," Tox21 Challenge to Build Predictive Models of Nuclear Receptor and Stress Response Pathways as Mediated by Exposure to Environmental Toxicants and Drugs, vol. 3, p. 78, 2017.

[12] R. Huang, M. Xia, D.-T. Nguyen, T. Zhao, S. Sakamuru, J. Zhao, S. A. Shahane, A. Rossoshek, and A. Simeonov, "Tox21challenge to build predictive models of nuclear receptor and stress response pathways as mediated by exposure to environmental chemicals and drugs," Frontiers in Environmental Science, vol. 3, p. 85, 2016. 
[13] A. Mayr, G. Klambauer, T. Unterthiner, and S. Hochreiter, "Deeptox: toxicity prediction using deep learning," Tox21 Challenge to Build Predictive Models of Nuclear Receptor and Stress Response Pathways as Mediated by Exposure to Environmental Toxicants and Drugs, p. 17, 2017.

[14] B. Ramsundar, S. Kearnes, P. Riley, D. Webster, D. Konerding, and V. Pande, "Massively multitask networks for drug discovery," arXiv preprint arXiv:1502.02072, 2015.

[15] G. E. Dahl, N. Jaitly, and R. Salakhutdinov, "Multi-task neural networks for qsar predictions," arXiv preprint arXiv:1406.1231, 2014.

[16] T. Unterthiner, A. Mayr, G. Klambauer, M. Steijaert, J. K. Wegner, H. Ceulemans, and S. Hochreiter, "Deep learning as an opportunity in virtual screening," in Proceedings of the Deep Learning Workshop at NIPS, 2014.

[17] I. Wallach, M. Dzamba, and A. Heifets, "Atomnet: a deep convolutional neural network for bioactivity prediction in structure-based drug discovery," arXiv preprint arXiv:1510.02855, 2015.

[18] A. Lusci, G. Pollastri, and P. Baldi, "Deep architectures and deep learning in chemoinformatics: the prediction of aqueous solubility for drug-like molecules," Journal of chemical information and modeling, vol. 53, no. 7, pp. 1563-1575, 2013.

[19] J. Ma, R. P. Sheridan, A. Liaw, G. E. Dahl, and V. Svetnik, "Deep neural nets as a method for quantitative structure-activity relationships," Journal of chemical information and modeling, vol. 55, no. 2, pp. 263-274, 2015 .

[20] D. K. Duvenaud, D. Maclaurin, J. Iparraguirre, R. Bombarell, T. Hirzel, A. Aspuru-Guzik, and R. P. Adams, "Convolutional networks on graphs for learning molecular fingerprints," in Advances in neural information processing systems, pp. 2224-2232, 2015.

[21] Z. Wu, B. Ramsundar, E. N. Feinberg, J. Gomes, C. Geniesse, A. S. Pappu, K. Leswing, and V. Pande, "Moleculenet: A benchmark for molecular machine learning," arXiv preprint arXiv:1703.00564, 2017.

[22] J. Gomes, B. Ramsundar, E. N. Feinberg, and V. S. Pande, "Atomic convolutional networks for predicting protein-ligand binding affinity," arXiv preprint arXiv:1703.10603, 2017.

[23] S. Kearnes, K. McCloskey, M. Berndl, V. Pande, and P. Riley, "Molecular graph convolutions: moving beyond fingerprints," Journal of computer-aided molecular design, vol. 30, no. 8, pp. 595-608, 2016.

[24] Z. Wu, B. Ramsundar, E. N. Feinberg, J. Gomes, C. Geniesse, A. S. Pappu, K. Leswing, and V. Pande, "Moleculenet: A benchmark for molecular machine learning," 2017.

[25] G. B. Goh, C. Siegel, A. Vishnu, N. O. Hodas, and N. Baker, "Chemception: A deep neural network with minimal chemistry knowledge matches the performance of expert-developed qsar/qspr models," arXiv preprint arXiv:1706.06689, 2017.

[26] D. Rogers and M. Hahn, "Extended-connectivity fingerprints," Journal of chemical information and modeling, vol. 50, no. 5, pp. 742-754, 2010.

[27] L. Ralaivola, S. J. Swamidass, H. Saigo, and P. Baldi, "Graph kernels for chemical informatics," Neural networks, vol. 18, no. 8, pp. 1093-1110, 2005.

[28] A. Bender, H. Y. Mussa, and R. C. Glen, "Screening for dihydrofolate reductase inhibitors using molprint $2 \mathrm{~d}$, a fast fragment-based method employing the naive bayesian classifier: limitations of the descriptor and the importance of balanced chemistry in training and test sets," Journal of biomolecular screening, vol. 10, no. 7, pp. 658-666, 2005.

[29] G. Schneider, W. Neidhart, T. Giller, and G. Schmid, "Scaffold-hopping by topological pharmacophore search: A contribution to virtual screening," Angewandte Chemie International Edition, vol. 38, no. 19, pp. 2894-2896, 1999.

[30] E. Gregori-Puigjané and J. Mestres, "Shed: Shannon entropy descriptors from topological feature distributions," Journal of chemical information and modeling, vol. 46, no. 4, pp. 1615-1622, 2006.

[31] G. Hinselmann, L. Rosenbaum, A. Jahn, N. Fechner, and A. Zell, "jcompoundmapper: An open source java library and command-line tool for chemical fingerprints," Journal of Cheminformatics, vol. 3, pp. 3-3, 2011.

[32] P. Mahé, L. Ralaivola, V. Stoven, and J.-P. Vert, "The pharmacophore kernel for virtual screening with support vector machines," Journal of Chemical Information and Modeling, vol. 46, no. 5, pp. 2003-2014, 2006.

[33] J. Alsenz and M. Kansy, "High throughput solubility measurement in drug discovery and development," Advanced drug delivery reviews, vol. 59, no. 7, pp. 546-567, 2007. 
[34] C. Steinbeck, Y. Han, S. Kuhn, O. Horlacher, E. Luttmann, and E. Willighagen, "The chemistry development kit (cdk): An open-source java library for chemo-and bioinformatics," Journal of chemical information and computer sciences, vol. 43, no. 2, pp. 493-500, 2003.

[35] T. Chen and C. Guestrin, "Xgboost: A scalable tree boosting system," in Proceedings of the 22Nd ACM SIGKDD International Conference on Knowledge Discovery and Data Mining, KDD '16, (New York, NY, USA), pp. 785-794, ACM, 2016.

[36] Y. LeCun, Y. Bengio, and G. Hinton, "Deep learning," Nature, pp. 436-444, May 2015.

[37] T. K. Ho, "Random decision forests," in Proc. Int. Conf. on Document Analysis and Recognition, pp. 278-282, 1995.

[38] A. Mayr, G. Klambauer, T. Unterthiner, and S. Hochreiter, "Deeptox: toxicity prediction using deep learning," Frontiers in Environmental Science, vol. 3, pp. 80

[39] S. J. Capuzzi, R. Politi, O. Isayev, S. Farag, and A. Tropsha, "Qsar modeling of tox21 challenge stress response and nuclear receptor signaling toxicity assays," Frontiers in Environmental Science, vol. 4, pp. 3 2296-665X, 2016.

[40] D. K. Duvenaud, D. Maclaurin, J. Iparraguirre, R. Bombarell, T. Hirzel, A. Aspuru-Guzik, and R. P. Adams, "Convolutional networks on graphs for learning molecular fingerprints," pp. 2224-2232, 2015.

[41] D. P. Kingma and J. L. Ba, "Adam: A method for stochastic optimization." arXiv Preprint [cs.LG]: 1412.6980, 2014.

[42] F. Chollet and et al, "Keras." https://github.com/keras-team/keras, 2015.

[43] M. Abadi, A. Agarwal, P. Barham, E. Brevdo, Z. Chen, C. Citro, G. S. Corrado, A. Davis, J. Dean, M. Devin, S. Ghemawat, I. Goodfellow, A. Harp, G. Irving, M. Isard, Y. Jia, R. Jozefowicz, L. Kaiser, M. Kudlur, J. Levenberg, D. Mané, R. Monga, S. Moore, D. Murray, C. Olah, M. Schuster, J. Shlens, B. Steiner, I. Sutskever, K. Talwar, P. Tucker, V. Vanhoucke, V. Vasudevan, F. Viégas, O. Vinyals, P. Warden, M. Wattenberg, M. Wicke, Y. Yu, and X. Zheng, "TensorFlow: Large-scale machine learning on heterogeneous systems," 2015. Software available from tensorflow.org.

[44] R. C. Glen, A. Bender, C. H. Arnby, L. Carlsson, S. Boyer, and J. Smith, "Circular fingerprints: flexible molecular descriptors with applications from physical chemistry to adme," IDrugs, vol. 9, no. 3, p. 199, 2006.

[45] G. Ridgeway, "Generalized boosted models: A guide to the gbm package," Update, vol. 1, no. 1, p. 2007, 2007.

[46] D. R. Koes, M. P. Baumgartner, and C. J. Camacho, "Lessons learned in empirical scoring with smina from the csar 2011 benchmarking exercise," Journal of Chemical Information and Modeling, vol. 53, pp. 1893-1904, 082013. 